\title{
Creación de redes globales a través de acciones locales: encuentros alternativos en la Educación Artística
}

\author{
Creation of global networks via local actions: alternative meetings \\ in Artistic Education
}

\author{
Teresa TORRES EÇA. InSEA (International Society for Education through Art). \\ teresatorreseca@gmail.com
}

Ana María BARBERO. Artista/Investigadora/Gestora Cultural. abarbero73@gmail.com

Resumen: Las TIC, por sus características propias (rapidez, interacción, globalidad), han permitido a los grupos sociales la creación de estructuras flexibles de organización y de negociación. Actualmente nuestras realidades humanas se expresan entre los espacios físicos y virtuales de comunicación. Cada uno de nosotros es parte de varias redes y habita encrucijadas multidimensionales. Las estructuras rizomáticas crecen en todas direcciones y las redes globales se construyen sin perder sus configuraciones individuales y locales. Los nuevos movimientos de código abierto proponen cohesión y el sincretismo. En estos movimientos, el respeto, la comprensión y el intercambio presentan una forma alternativa de pensar y de comportarse, desafiando a los sistemas capitalistas neoliberales. El factor clave para mantener las redes es la sinergia. Las organizaciones dedicadas la educación artística, así como los individuos interesados en el tema, pueden trabajar de forma conjunta, a través de las diversas redes, como la Alianza Mundial para las Artes (WWA). Como profesores e investigadores debemos fomentar el uso de estas herramientas a través de las redes, favoreciendo el surgimiento de una inteligencia colectiva capaz de transformar la sociedad en nuestros campos interconectados de la educación y las artes. Para ello necesitamos hojas de ruta, agendas, días conmemorativos y declaraciones globales, aunque tales herramientas carecerán de valor si las estructuras individuales y locales no participan en su construcción mediante el intercambio de sus experiencias a una escala global. 
Palabras clave: sostenibilidad; activismo; red; educación artística

"We live in the lap of an immense intelligence. But that intelligence is dormant and its communications are broken, inarticulate and faint until it possesses the local community as its medium."

John Dewey(In: Kadlec, 2007)! .

\begin{abstract}
For their own characteristics (speed, interaction, global) ICT have allowed social groups creating flexible structures and organizational negotiation. Currently our human realities are expressed between physical and virtual spaces of communication. Each of us is part of several networks and multidimensional crossroads. Rhizomatic structures grow in all directions and global networks are built without losing their individual and local configurations. The new open source movements proposed cohesion and syncretism. In these movements, respect, comprehension and exchange present an alternative of thinking and behaving, challenging the systems forms of capitalism. The key factor to keep networks is synergy. Organizations dedicated to arts education, as well as individuals interested, can work together, through various networks such as the World Alliance for the Arts (WWA). As teachers and researchers we should encourage the use of these tools through networks, favoring the emergence of collective intelligence and transforming our interconnected society in the fields of education and arts. For this, we need roadmaps, diaries, memorial days and global declarations, although such tools have no value if the individual and local structures are not involved in its construction by sharing their experiences on a global scale.
\end{abstract}

Keywords: sustainability; activism; network; arts education.

\title{
Las interacciones sociales
}

Pierre Levy (1994) nos alerta del paso de una economía basada en la información a una economía basada en las interacciones humanas: una economía social. Para Levy, nuestra humanidad es lo más preciado que tenemos. Nuestras realidades humanas se expresan, en la actualidad, entre los espacios físicos y virtuales de comunicación. Después del primer "encantamiento" con el nuevo y esplendoroso mundo cibernético y del gran temor al teatro post-global, después de las premoniciones de McLuhan, ahora estamos reflexionando sobre las formas en las que la democracia participativa puede existir a través de nuestras experiencias de conectividad globales.

The more you create village conditions, the more discontinuity and division and diversity. The global village absolutely insures maximal disagreement on all

1. Traducción Propia: "Vivimos en el regazo de una inteligencia inmensa. Pero esta inteligencia se encuentra dormida y sus comunicaciones están rotas, inarticuladas y débiles hasta que posea a la comunidad local como su medio. " John Dewey (En: Kadlec, 2007). 
points. It never occurred to me that uniformity and tranquility were the properties of the global village. It has more spite and envy. The spaces and times are pulled out from between people. A world in which people encounter each other in depth all the time. The tribal-global village is far more divisive - full of fighting than any nationalism ever was. Village is fission, not fusion, in depth all the time. (McLuhan, 1996. From the "The Playboy Interview")2

En el texto citado en líneas superiores, que fue extraído de una entrevista de Playboy (1969) a McLuhan, este visionario, creador de la expresión la Aldea Global, reflexiona sobre la esencia misma de este concepto, de cómo él lo entiende, expresando su creencia sobre las condiciones de la aldea global y comentando que "la aldea global hace que el máximo desacuerdo y diálogo creativo sean inevitables." Y lo cierto es que, hoy en día, estamos viviendo estas divisiones con una necesidad especial de diálogos creativos.

\section{La Inteligencia Colectiva}

Desde nuestro punto de vista, un concepto clave en el mantenimiento de la red contra esta división, competitividad y fragmentación de la que nos habla McLuhan, es la creación de sinergias basadas en los valores de la cohesión, la colaboración y la síntesis. La selección de la información es crucial para sobrevivir. Los Nuevos Medios, o no tan nuevos, ofrecen oportunidades rápidas, interactivas y accesibles para casi todos los individuos y grupos en la sociedad.

Las formas novedosas de creación de grupos, el intercambio de conocimientos y experiencias están emergiendo rápidamente. Cada vez más, tenemos la capacidad de compartir información en forma de imágenes, texto y sonido. De este modo, los individuos, las comunidades y los grupos se ven confrontados con las costumbres, creencias, esperanzas y miedos de los demás. Este conjunto de grupos locales funcionan como estructuras rizomáticas, repartidas en todas las direcciones, y permiten formar redes mundiales a través de nodos de conexión. La forma en la que estos grupos están procesando, compartiendo y entremezclando la información, es decir, la creación de nuevo conocimiento podría ser visto como una forma de inteligencia colectiva (Levy, 2004). Según Fitzgerald (2005), la inteligencia colectiva se expresa en la medida que los grupos, a menudo, pueden pensar más que los expertos de forma individual, actuando como motores paralelos en el

2. Traducción Propia: Cuantas más condiciones creas para un pueblo, mayor es la discontinuidad y de la división y la diversidad. La aldea global asegura absolutamente el desacuerdo máximo en todos los puntos. Nunca se me ocurrió que la uniformidad y la tranquilidad eran las propiedades de la aldea global. Cuenta con más rencor y con la envidia. Los espacios y los tiempos son tirados de entre las personas. Un mundo en el que las personas se enfrentan entre sí todo el tiempo. La aldeatribal global es mucho más divisoria - llena de combates - de lo que lo fue cualquier nacionalismo alguna vez. El Pueblo es la fisión en profundidad, no la fusión, todo el tiempo. Texto extraído de The Playboy Interview: MarshallMcLuhan”, Playboy Magazine, Marzo 1969 último acceso a 12/8/13. Retirado de http://www.nextnature.net/2009/12/the-playboy-interviewmarshall-mcluhan/. 
procesamiento de las decisiones, poniendo en marcha este conocimiento colectivo para responder incluso a las preguntas mas difíciles. En este tipo de inteligencia colectiva corta (short collective intelligence) existe la idea de que un grupo puede ser más inteligente que sus miembros. La inteligencia de esta forma será colectiva, porque surge de las interacciones entre millones de individuos.

\section{Las Redes}

Con fecha de 11 agosto 2013 hicimos una prueba: colocamos en el buscador de Google la expresión 'art education network', seleccionamos en herramientas la opción 'todos lo idiomas' para ampliar nuestra búsqueda al máximo posible, y obtuvimos un resultado de 833.000 similitudes en 0,12 segundos. Estos datos pueden darnos una pequeña idea sobre cuántos grupos estaban discutiendo la educación artística en el mundo en aquel momento, o sobre los grupos que incluyen intereses profesionales vinculados con la enseñanza del arte. Pero lo que no nos dan a entender son las conexiones que se establecen entre ellos y cómo dichas conexiones están generando conocimiento. Sin embargo, es precisamente la calidad de esas conexiones o las interacciones sociales que se establecen entre ellas, lo que realmente nos interesa en términos de encuentros útiles para los educadores de arte. Lo que queremos apuntar con los datos anteriores es que, a pesar de todos los avances existentes en la creación de redes y bases de datos, todavía no podemos crearnos una imagen clara de las redes dedicadas a la educación artística y su funcionamiento real, dado que ni siquiera conseguimos entender las conexiones reales que se establecen entre ellas. La realidad es que, a pesar de todos los avances que alcanzados, todavía existe mucho trabajo por hacer con el fin de beneficiarnos al máximo de los Nuevos Medios.

Como profesores o investigadores de la educación, lo que nos interesa es trabajar con herramientas de transformación, por lo que la inteligencia colectiva que surge de las herramientas de comunicación accesibles a través de las tecnologías digitales puede ser una vía, una posibilidad, de transformar la sociedad en nuestros campos interconectados de la pedagogía y el arte. Desde el punto de vista de la creación de redes, los diálogos visibles y la acción son algunas de las posibilidades que emergen de las actividades pedagógicas/artísticas como actos políticos. Políticos, en el sentido de que cada acto de transformación educativa tiene bases políticas (Giroux,1988). Pero, para obtener la visibilidad que necesitamos, es necesaria una mayor conectividad entre la información, lo que no siempre es fácil de obtener. Tal vez este sea una de las áreas por explorar más urgentes, en materia de investigación, para aquellos equipos interdisciplinarios de investigadores en los campos de la educación en las artes y las tecnologías digitales.

\section{Múltiples encrucijadas}


A medida que nos acercamos a las múltiples encrucijadas, que se presentan en la educación artística, a través de las diversas relaciones y conexiones posibles, es más importante parar y reflexionar sobre ellas como si de un sistema inteligente y complejo se tratase. Un sistema formado por algunos valores complementarios que, desde la perspectiva de una inteligencia cooperativa o a través de las lentes de una inteligencia cívica, se traducen en la capacidad de los grupos y organizaciones en su conjunto para diseñar y aplicar estrategias eficaces, equitativas y sostenibles a los problemas comunes (Schuler, 2008). Aquí, cada individuo tiene algo que aportar, y el valor de cada contribución solo puede apreciarse integrado en un inteligencia final constituida por las aportaciones de todos. Pero más que reconocer cómo funciona el sistema tenemos que entender cómo podemos utilizarlo como una herramienta de transformación para una democracia participativa efectiva. Estas herramientas de transformación son definidas por Freire (1970) como herramientas que pueden empoderar a las comunidades y a los particulares a través de la construcción conjunta del aprendizaje. Algo que se hace evidente en las redes, donde muchos usuarios sienten un despertar hacia situaciones colectivas en grupos sociales como Facebook , Myspace, World Cyber Cafes, Diáspora, Second Life o, incluso, en los juegos de rol (RPG y/o MRPG), pero el hecho de que se establezcan estas conversaciones colectivas no significa que Internet sea la revitalización de las prácticas democráticas. Sin embargo, podemos decir que cada vez más se puede observar la distribución en Internet de contenidos diversos (audio, visual o audiovisual) relacionados con la justicia social, el activismo y la acción comprometida con el desarrollo sostenible. Poco a poco movimientos en pro de estos valores están emergiendo después de un período de relativa pérdida de fe en el futuro:

\section{Re-claim the future!}

...It is increasingly clear that our 21 st century challenges can no longer be met with 20th century approaches, including how we think about the future. There is a pressing need to broker much wider links between the arts and those working in sustainability to create a new 'community of practice'. It is only when we join the dots that we can really begin to think differently - and doing this can help us access tools, technologies and techniques that enable us to imagine what it would be like to live and love in a world were we have risen to our global challenges, and transformed our anguish into empowerment. ${ }^{3}$

\section{Zero Carbon Britain: Rethinking the Future, p.139}

Este pequeño fragmento ha sido recuperado de un informe publicado en 2013 por investigadores del Proyecto Carbon Cero, en Inglaterra, que ilustra un punto de inflexión definitivo en nuestras sociedades. Hay una necesidad urgente de dar a conocer las amenazas reales de nuestra naturaleza humana y planetaria y como no han habido respuestas o responsabilidades adoptadas por las instituciones oficiales ante los desastres ecológicos reales, más y más personas de todas las áreas de las ciencias y las humanidades empiezan a pensar su función educativa de una forma 
mucho más activista y desde una perspectiva más comprometida. En el Proyecto Carbon Cero, el Centro de Tecnología Alternativa pretende reunir gentes de un amplio espectro de diferentes disciplinas con el objetivo de informar, inspirar y permitir a la sociedad contemporánea adoptar los cambios necesarios para repensar el futuro, para mostrar cómo se puede hacer frente al desafío climático sin depender en las promesas de la tecnología del futuro usando sólo lo que existe ahora, es decir, usando los medios disponibles para ser implementados inmediatamente.

La necesidad de llevar a cabo pequeñas acciones de carácter local, va a la par de una concienciación cada vez mayor de las personas, que adquieren esta consciencia a través de las redes sociales, donde la información se transmite rápidamente provocando la necesidad de acciones sostenidas y sustentables en el tiempo. Muchos artistas, científicos y educadores de arte se encuentran en el primer lugar de estos movimientos alternativos y, por medio de la conexión que existe entre ellos, están creando redes globales formadas por aquellas personas interesadas en realizar acciones locales para preservar los pocos patrimonios naturales o culturales tangibles e intangibles que quedan.

Por ejemplo, la joven diseñadora, educadora e investigadora Angela Saldaña, que está llevando a cabo un estudio de investigación activista de la pequeña comunidad de su familia (comenzó su estudio con narraciones de cuentos sobre la vida de su abuela paterna y sus amigos en un pueblo cerca de la costa norte de Portugal) poco a poco comenzó a sentir que como investigadora podía hacer mucho más a través del trabajo colaborativo con los participantes: siete personas de edad y sus familias, grupos de interés de la comunidad, los artistas locales y no locales. Juntos, a través de los recuerdos y las representaciones de la memoria, revitalizan las tradiciones sociales y las nuevas formas de generar diálogos intergeneracionales, mediante el desarrollo de proyectos relacionados con el arte contemporáneo y los medios de comunicación.

Los valores que primaron en el desarrollo de estos procesos son el respeto, la comprensión y el intercambio, valores sin duda de la educación a través del arte. Estos valores son en realidad un desafío a las ortodoxias políticas y culturales actuales. Lo cierto es que, las personas y grupos involucrados en la educación artística pueden trabajar juntos para crear redes locales, es decir, para crear estructuras desarrolladas a partir de la acción local.

Estamos firmemente convencidos de que a fortaleza futura de la educación artística

3. Traducción Propia: Reclama el futuro! (...) Cada vez está más claro que los desafíos del siglo 21 ya no se pueden cumplir con los enfoques del siglo 20, incluyendo la forma en que pensamos sobre el futuro. Hay una necesidad urgente de negociar vínculos mucho más amplios entre las artes y los que trabajan en la sostenibilidad para crear una nueva "comunidad de práctica". Es sólo cuando unimos los (diferentes) puntos cuando realmente podemos empezar a pensar de manera diferente y para hacer esto nos pueden ayudar las herramientas de acceso, tecnologías y técnicas ya que nos permiten imaginar lo que podría ser vivir y amar en un mundo donde hayamos alcanzado nuestros desafíos globales y transformado nuestra angustia en empoderamiento. Zero Carbon Britain: Rethinking the Future, p.139. 
dependerá no sólo de las actividades de las estructuras locales, sino también de la forma en que son visibles, provocando impactos en distintas redes geográficas y culturales. Podemos pensar que tenemos agendas, estrategias, días conmemorativos y mapas de carreteras, pero todo lo anterior no será más que un callejón sin salida si los grupos locales y los individuos no participan en el proceso constructivo, compartiendo la experiencia local a un escala global a través de diferentes portales.

Otro ejemplo similar al anterior lo encontramos en el proyecto desarrollado en Tondela (Viseu, Portugal) por el artista cubano y activista político, Yuraldi Rodriguez Puentes, AL-Tondela. Un proyecto escultórico que partió de una leyenda local pero que integró la participación en el mismo de más de doscientas personas. En este caso, además de la recuperación de las tradiciones locales, el tema de trabajo fue reflexionar sobre el papel de las mujeres en las comunidades locales. A través de visitas durante la ejecución del proyecto y mediante la inclusión en la pieza de pequeñas piezas realizadas por niños, jóvenes, profesores y otros artistas, el escultor consiguió crear un proyecto pedagógico artístico, de carácter social y colaborativo que a su vez surgió de un otro proyecto comunitario desarrollado en la pequeña aldea de SoutoBom, en Tondela. Cabe aquí decir que este proyecto sólo fue posible gracias al apoyo del Instituto Piaget de Viseu, que cedió un espacio (un anfiteatro) al escultor para la ejecución de su obra. Como contrapartida, el artista se ofreció a tener el taller abierto en determinadas horas para explicar su proyecto tanto a los funcionarios de la institución, como a las visitas de escuelas y otras entidades que fueron organizadas con tales fines. Señalar aquí, que este tipo de colaboraciones son importantísimas para cultivar ese gusto por las artes que surge desde la experiencia, la curiosidad y el conocimiento.

El proyecto "Os Ambientes do Ar", comunitario centrado en la salvaguarda y valorización del patrimonio local resultado de un proyecto de investigación iniciado en el año 2001, en la Aldea de Souto Bom, con la organización del 1er. CAMPO EUROPEO DEL PATRIMONIO en la aldea. Este Campo del Patrimonio reunió en Souto Bom a estudiantes de arquitectura, de artes, de historia, y de otras áreas junto con especialistas en diversas materias como biología, geología, arquitectura, etnografía, etc.

Durante los quince días que duró fueron realizados diversos inventarios del patrimonio construido de Souto Bom, se realizaron diversas entrevistas a las gentes de la aldea, se estudiaron los molinos, la fauna y la flora de la ribera de la Pena y se organizaron diversas actividades y conferencias. Tomando como referencia el saber (know how) de las gentes de Souto Bom, el paisaje y un conjunto de pequeños 


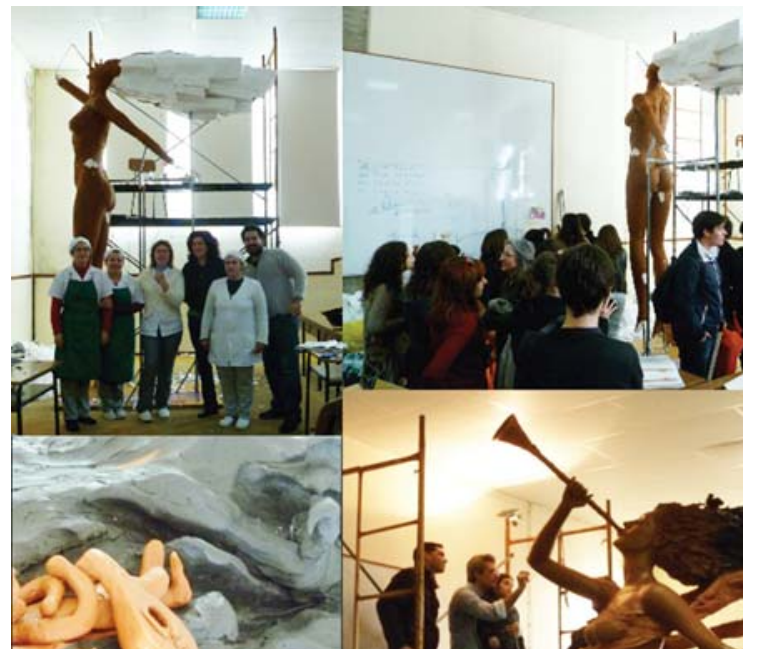

Fig. 1. En el taller (Fuente: Imágenes cedidas por el escultor $)^{4}$.

molinos de rueda horizontal situados en los márgenes de la ribera de la Pena, se creó un proyecto cultural con el doble objetivo de salvaguardar la cultural y el patrimonio del lugar y potenciar un desarrollo local sostenible y a escala humana ${ }^{6}$.

Este proyecto, coordinado por la artista e investigadora Ana María Barbero, surgió como proyecto del Forum UNESCO: Universidad y Patrimonio -otra de las grandes

Fig. 2: Participantes en el Campo Europeo del Patrimonio, frente a entrada del Molino n. ${ }^{\circ}$ 7. (Fuente: Proyecto Os Ambientes do Ar, A.B.).

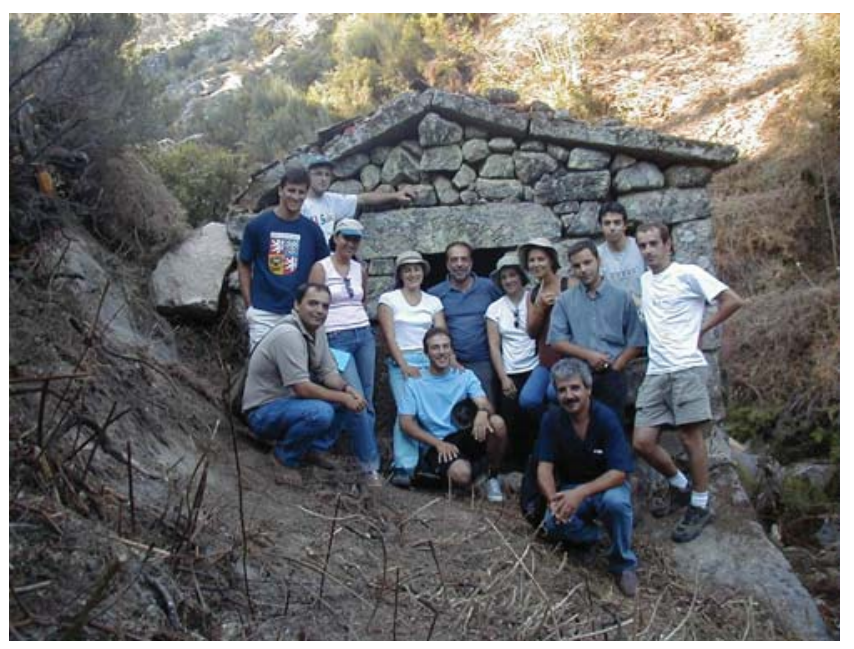

4. Es posible ver mas obra de Yuraldi Rodriguez Puentes en Facebook (https://www.facebook.com/yuraldi.puentes?fref=ts) 5. Barbero, A. (2011): La Gestión del Patrimonio Histórico como Instrumento para un desarrollo Sostenible. Un caso práctico: El proyecto de Desarrollo Local “Os Ambintes do Ar”. Col. Vítor, 280. Ediciones Universidad de Salamanca. Para mas información sobre este proyecto consultar también la página web http://soutobom.wix.com/os-ambientes-do-ar 
redes de trabajo universitarias relacionadas con la educación patrimonial- con sede en el Instituto Piaget de Viseu, en Portugal. Comenzó siendo un inventario del patrimonio local (material e inmaterial) y terminó dando origen a un tesis de doctorado, a múltiples artículos, vídeos, una página web, a dos libros educativos, fichas diversas de trabajo y a un proyecto real de desarrollo local. Comentábamos antes la necesidad de estar presentes en las redes, por ello, podemos encontrar información sobre este proyecto en la página web, en facebook y en youtube. Por ejemplo, si alguien quisiera conocer algo más sobre la componente educativa, puede hacerlo vía Ambientes do Ar Novo, en youtube https://www.youtube.com/ watch? $=4$ WSXKDp-pVw

Además de estos ejemplos, podemos citar otros investigadores de la educación artística que están construyendo datos empíricos sobre la forma en la que los proyectos de educación artística son desarrollados en las comunidades, las escuelas y las universidades con el fin de conseguir entornos más sostenibles. Algunas de esas experiencias se reportan en revistas especializadas como la International Journal of Education Through Art (la Revista Internacional de la Educación a través del arte) o la International Journal of Art and Design Education (Revista Internacional de Arte y Diseño). Sólo para nombrar unos pocos proyectos vamos a mencionar dos artículos publicados en el volumen 8 , número 3 de la la primera: 'Berry wars: A science centre as a forum for a dialogical activist, interdisciplinary art project' (Las

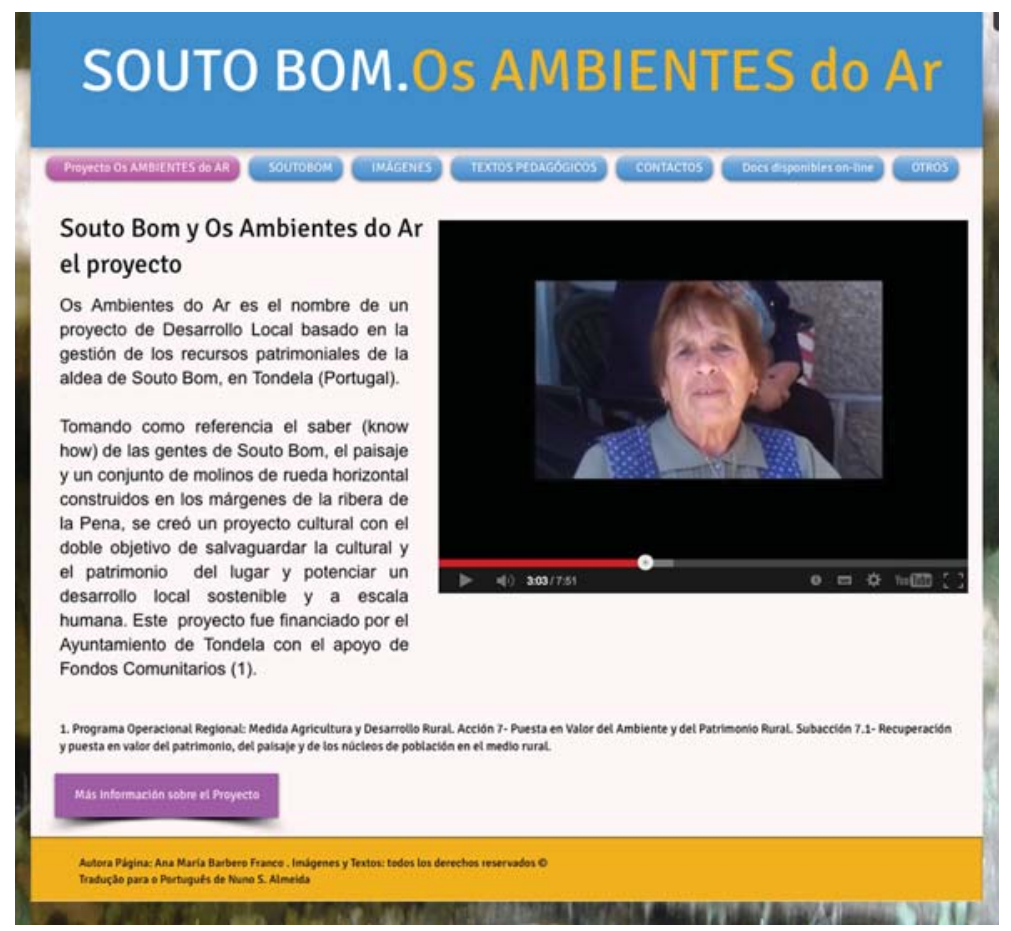

Fig. 3 Página Web de SoutoBom (Fuente: http://soutobom.wix.com/ os-ambientes-do-ar\#!videos/c16x4 )

6. Fue financiado por el Ayuntamiento de Tondela con el apoyo de Fondos Comunitarios, Programa Operacional Regional: Medida Agricultura y Desarrollo Rural. Acción 7- Puesta en Valor del Ambiente y del Patrimonio Rural, Sub-acción 7.1Recuperación y puesta en valor del patrimonio, del paisaje y de los núcleos de población en el medio rural. 

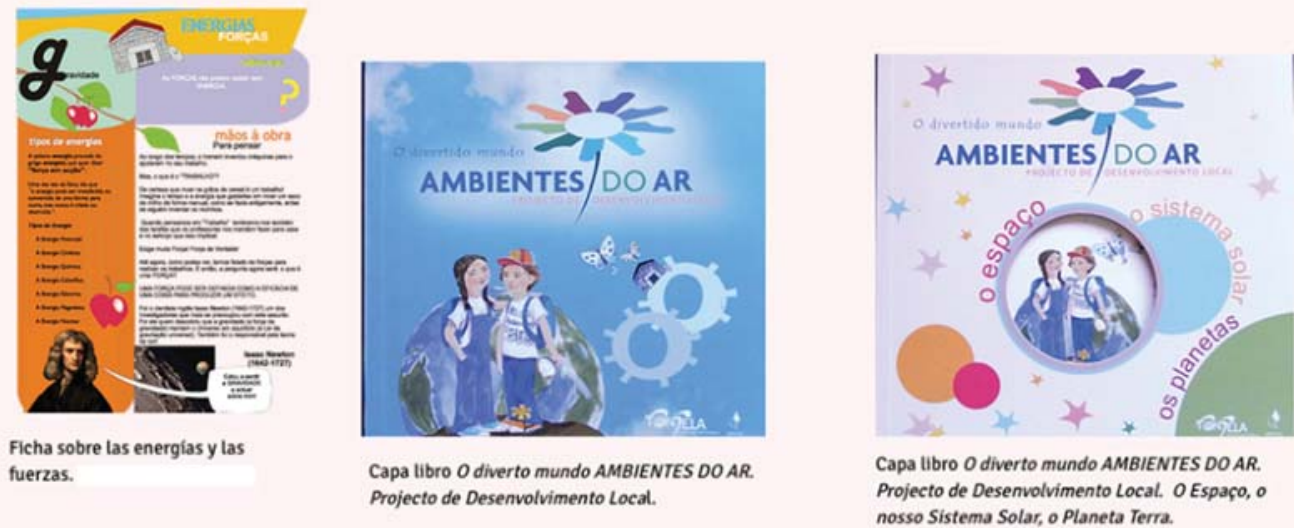

Capa libro $O$ diverto mUndo AMBIENTES DO AR. Projecto de Desenvolvimento Local. OEspaço, o nosso Sistema Solar, o Planeta Terra.

Fig. 4. Portadas de algunos materiales pedagógicos del proyecto Os Ambientes do Ar. (Fuente: http://soutobom.wix.com/os-ambientes-do-ar\#!videos/c16x4)

guerras de Berry: un centro de la ciencia como foro para un activista dialógico, un proyecto de arte interdisciplinario), por Maria Huhmarniemi y 'Catch and release: Artworks inspiring insight into environmental issues' (Captura y liberación: Obras de Arte que inspiran visiones sobre temas ambientales) por Ruth Cerveza y Kit Grauer .

El proyecto de Berry consistió en una colaboración entre artistas, activistas, investigadores y divulgadores científicos que se centraron en la industria de la baya Lapish y en la relación existente entre los recolectores de estas bayas (esta industria había visto un creciente número de recolectores de bayas de temporada extranjeros que llegaban a los bosques finlandeses dando lugar a conflictos entre los recolectores de bayas locales y extranjeros). El objetivo del proyecto era crear un diálogo entre los recolectores de bayas extranjeros y lugareños hacia la comprensión de la riqueza de la cultura de la recolección de bayas en Finlandia (Huhmarniemi, pp 287-303 ). Catch and Releas es, en definitiva, una investigación relacionada con un proyecto, financiado por el gobierno de Canadá, consistente en la producción de nuevas obras de arte interactivas por un equipo formado por artistas, educadores y diseñadores en colaboración con el centro de interpretación situado en la histórica fábrica de latas de conserva localizada en el Parque Nacional del Golfo de Georgia.

La exposición y actividades educativas realizadas, estaban encaminadas a fomentar el conocimiento de la historia social de la región y las condiciones culturales contemporáneas - 'capturando y liberando' historias en la esfera pública (Beer \& Grauer, pp 349-360) .

El impacto causado por todos estos proyectos a corto y largo plazo sobre las comunidades y en sus círculos de investigación regional, se ha expandido al hacerlos visibles a través de su publicación en diversas revistas de carácter internacional, como es el caso de la revista de la Sociedad Internacional para la Educación a 
través del Arte (InSEA International Society for Education through Art). Gracias a estas publicaciones, y a su divulgación a través de las redes, otros educadores del arte de todo el mundo pueden sentirse alentados a perseguir objetivos artísticos, comunitarios o ambientales similares, diseñando así nuevos paradigmas para la educación artística.

Hasta ahora, las revistas académicas internacionales son casi las únicas herramientas de comunicación para difundir estas experiencias. Podemos, sin embargo, reclamar otros medios. Lo cierto es, que estaríamos ofreciendo un pobre servicio a la comunidad educativa si no utilizáramos todos los medios disponibles a nuestro alcance para crecer y hacer visibles nuestras acciones de una manera crítica. Cada vez son más las voces que están apareciendo desde todo tipo de campos que reclaman una cultura libre, el conocimiento de código abierto y los materiales educativos de código abierto (OER), que pueden utilizar tecnologías de comunicación existentes para reconstruir las prácticas democráticas de comunicación para un futuro más sostenible. Los educadores de arte contemporáneo no están fuera de esta corriente, muchos de ellos están utilizando pedagogías creativas en proyectos multidisciplinarios locales a través de asociaciones para nutrir el desarrollo sostenible, la solidaridad mundial, la cooperación y los valores de derechos humanos. Todos ellos están tratando de hacer visibles este tipo de proyectos que contribuyen a una sociedad basada en el conocimiento, pero tal vez esto no sea suficiente y debamos ayudarles a expandir esta información mucho mas a través de nuestras conexiones.

\section{Portales como Puertas de Enlace}

El Conectivismo es la tesis de defiende que el conocimiento se distribuye a través de una red de conexiones, por lo que el aprendizaje consiste en la capacidad de construir y atravesar esas redes. (Downes, 2012).

Existen en la actualidad varias plataformas que se utilizan como conexión para entornos profesionales y para entornos educativos. Para autores como Stephan Downes: 'learning is the creation and removal of connections between the entities, or the adjustment of the strengths of those connections' (Downes, 2012, p.9) ${ }^{7}$. Durante los últimos veinticinco años, la tecnología ha cambiado la forma cómo nos comunicamos y cómo aprendemos. El aprendizaje parece ser más un reflejo de los ambientes sociales subyacentes. Vaill (1996) hace hincapié en que el aprendizaje debe ser una forma de ser, "an ongoing set of attitudes and actions by individuals and groups that they employ to try to keep abreast the surprising, novel, messy, obtrusive, recurring events (... ) (p.42) .

Por otro lado, concordamos con Siemens (2004) cuando afirma que el campo de la

7. Traducción Propia: "el aprendizaje es la creación y la eliminación de las conexiones entre las entidades, o el ajuste de los puntos fuertes de esas conexiones" (Downes, 2012, p.9). 
educación ha sido lenta en reconocer tanto el impacto de las nuevas herramientas de aprendizaje como los cambios ambientales en lo que significa aprender. Como profesionales de la educación artística a menudo carecemos de habilidades o recursos para trabajar con las herramientas de conexión disponibles. Nuestra forma de trabajar ha sido alterada por las nuevas herramientas de Internet que estamos utilizando actualmente. Estas tecnologías son necesarias para lograr esa economía social prevista por Pierre Levy (1994). Las formas de trabajo son diversas, y sin lugar a duda, el trabajo colaborativo es una de ellas. En cuanto a los educadores de arte, muchos grupos y millones de bytes de información son accesibles, pero la construcción del conocimiento efectivo a través de las redes de conexión, se encuentra todavía en una etapa embrionaria. Desde un punto de vista operativo, se debe poner un mayor esfuerzo en la creación de equipos interdisciplinarios dedicados al desarrollo de los portales de comunicación digital, con el fin de proporcionar más oportunidades de aprendizaje a las personas que normalmente no tienen acceso a la información.

\section{WAAE}

La Alianza Mundial para la Educación Artística (WAAE ) fue creada en el año 2006, para preparar un discurso estratégico para la primera Conferencia Mundial de la UNESCO sobre la Educación Artística que tuvo lugar en Lisboa, Portugal, en marzo de 2006. Antes de la conferencia de la UNESCO, los presidentes, por aquel entonces, de la Asociación Internacional de Drama/ Teatro y Educación (IDEA) Dan Baron, de la Sociedad Internacional para la Educación Musical (ISME) - Gary McPherson, y de la Sociedad Internacional para la Educación por el Arte (InSEA) - Doug Bougton, se reunieron durante 5 días (del 1 al 5 marzo) en Viseu, Portugal, como conferencistas invitados al congreso Mundial de InSEA2006. La organización de esta Alianza, la idea de esta reunión, salió de los miembros de la ejecutiva de InSEA, Diederik Schonau y John Steers. Se pensaba que las grandes organizaciones de educación artística debían cooperar para destacar el papel de la educación artística en el mundo, y esta visión dio resultados fructíferos. Uno de ellos, fue la declaración conjunta que influyó en los debates de las tres organizaciones durante el evento de la UNESCO:

For more than half a century, our associations have contributed significantly o the development of curricula and teaching approaches. We are now ready to respond proactively to the diverse social and cultural needs of our world...to collaborate with all governments, networks, institutions, communities and individuals who share our vision.

The Joint Declaration, WWA, $2006^{9}$

\footnotetext{
8. Traducción Propia: "un conjunto permanente de actitudes y acciones de los individuos y los grupos que emplean para tratar de mantenerse al tanto de los sorprendentes, nuevos, desordenados, entrometidos, hechos recurrentes (...)". Traducción Propia: Durante más de medio siglo, nuestras asociaciones han contribuido de manera significativa aldesarrollo de planes de estudios y métodos de enseñanza. Ahora estamos listos para responder de manera proactiva a las diversas necesidades sociales y culturales de nuestro mundo ... para colaborar con todos los gobiernos, las redes, las instituciones, las comunidades y los individuos que comparten nuestra visión. La Declaración Conjunta, WWA, 2006.
} 
En el 2008, la Alianza Mundial para la Danza se unió a la WWA, y las cuatro organizaciones han trabajado, desde entonces, por una mayor cooperación y una mayor visibilidad, en especial en los ámbitos de los debates de la UNESCO, lanzando tres grupos de trabajo estratégicos en la investigación, la creación de redes y la defensa de las artes. Es interesante ver cómo el trabajo en red se convierte en una gran preocupación para estas instituciones, preocupadas también por encontrar espacios físicos para el desarrollo de los debates en lugares públicos (para dar así una mayor visibilidad a la educación artística). Estos debates toman la forma de conferencias o congresos y se organizan siempre en colaboración con otras organizaciones como universidades, gobiernos y fundaciones. Ponemos el ejemplo de la Conferencia WAAE 2009 que fue organizada en colaboración con la asociación Creativity, Culture and Education (UK) y la Newcastle Gateshead Initiative, en Newcastle, Reino Unido. En esta conferencia fueron preparadas las recomendaciones estratégicas para que una delegación de la WAAE las presentara en la UNESCO, en París, en noviembre de 2009. Estas recomendaciones también fueron presentadas en la Segunda Conferencia Mundial de la UNESCO sobre la Educación Artística, en Seúl, Corea del Sur, en mayo de 2010.

Otra demostración del poder de redes de WWA fue la conferencia WAAE 2012 en Rovainiemi, organizada por la Universidad de Laponia, Finlandia, en asociación con el Instituto de Cultura del Norte, y la conferencia WAAE 2013, en mayo 2013, organizada por la Universidad de Erlangen- Nuremberg en colaboración con la Federación alemana para la Educación de las Artes y la Fundación alemana Stiftung Mercator. Todas estas redes de trabajo fueron muy positivas e hicieron posible la concreción de importantes colaboraciones en términos de promoción de la educación artística, pero desde nuestro punto de vista, hubo un aspecto que no fue bien tratado: la construcción de una red sólida en la esfera virtual para mantener y ampliar los debates entre las organizaciones y la visibilidad de la investigación y práctica en la educación artística. Por supuesto, las conferencias de alto nivel y las reuniones de expertos son importantes, pero, su significado está en riesgo cuando lo que sostiene la educación artística, que es la práctica, no está conectado y valorado a través de la visibilidad de las redes de trabajo. Si no logramos unir las redes, no seremos capaces de lograr las creación de nuevos conocimientos de forma colaborativa y, por lo tanto, será difícil seguir adelante en la comprensión y defensa de la educación artística.

\section{Acciones locales - Redes locales}

Una de nuestras propuestas, es precisamente que las organizaciones centradas en la educación artística, y las personas interesadas en esta área, pueden y deben trabajar juntos en la creación de estructuras locales, con contenido, es decir, estructuras construidas a base de acciones reales. Las fortalezas y debilidades de la educación artística futuras dependerán de las acciones desarrolladas por estas estructuras, en la forma en que se distribuirán y divulgarán en la red y en el impacto de sus productos 
en las distintas redes geográficas y culturales. No obstante, continuamos creyendo necesarias hojas de ruta; agendas; días conmemorativos y orientaciones globales, aunque dichas herramientas carecerán de valor, como ya hemos indicado con anterioridad, si las estructuras individuales locales no participan en su construcción mediante el intercambio de experiencias locales a una escala global.

En ese sentido, la acción de la convocatoria para la celebración de la Semana Internacional de la Educación Artística lanzada por la UNESCO en el año 2012, bajo la recomendación de representantes de la WWA, era indicativo de las posibilidades de creación de redes locales. De esta forma, no solo se celebró en 2012 y 2013 de manera formal a través de los canales de la UNESCO, sino que también fue celebrada por los pequeños grupos de educadores de arte que respondieron a la llamada de InSEA, en los distintos grupos de educación en el arte, vinculados a través de foros

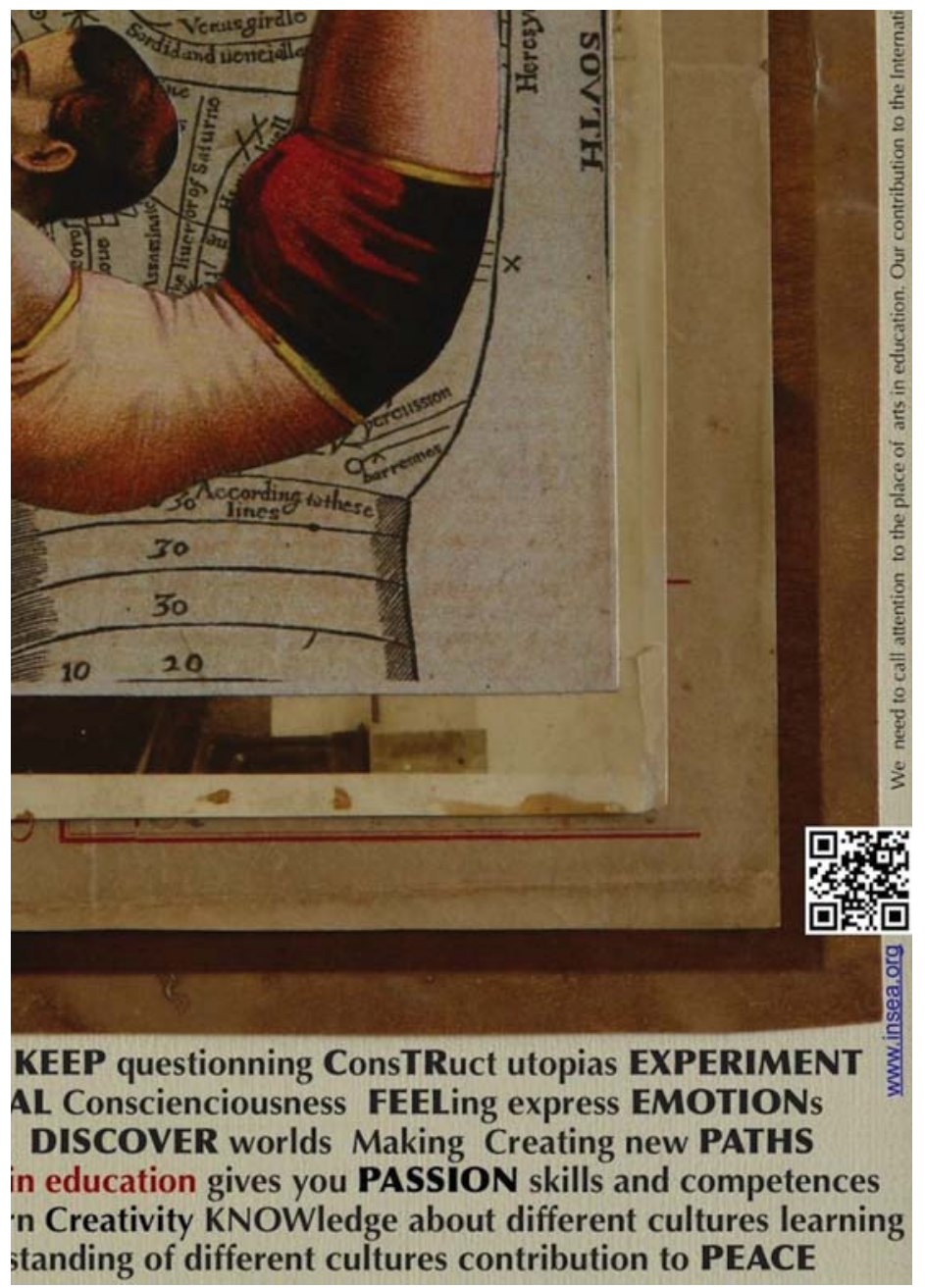

Fig. 5: Postal para la acción "más que nunca" enviado a través de las redes sociales durante la primera semana de la educación artística internacional, diseñada por el grupo C3, en Santiago de Compostela, España 
Torres \& Barbero / Creación de redes globales a través de acciones locales: Encuentros alternativos en la Educación Artística

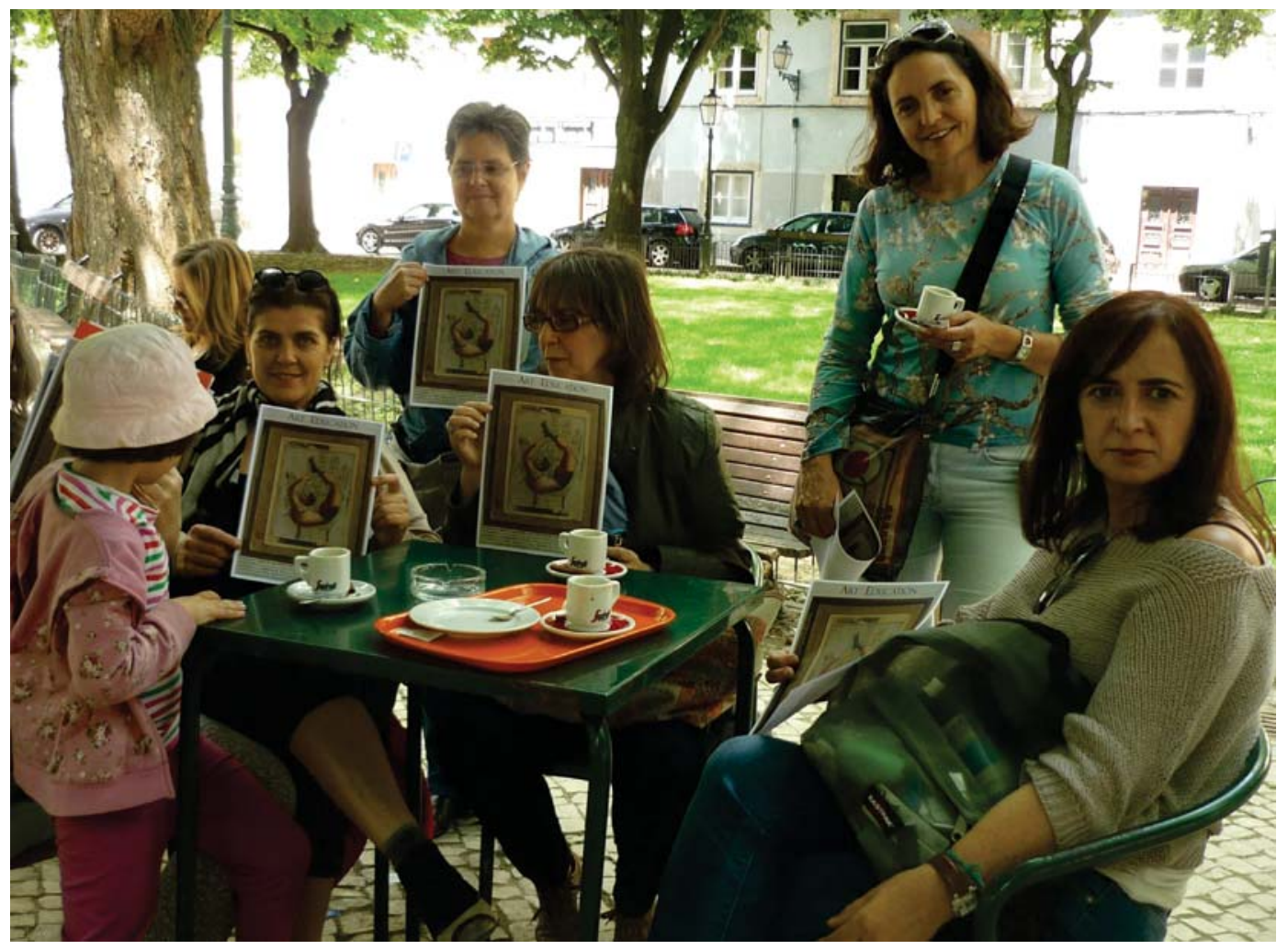

Fig. 6: Postal de "más que nunca" en Lisboa, Portugal. Fuente: T.E.

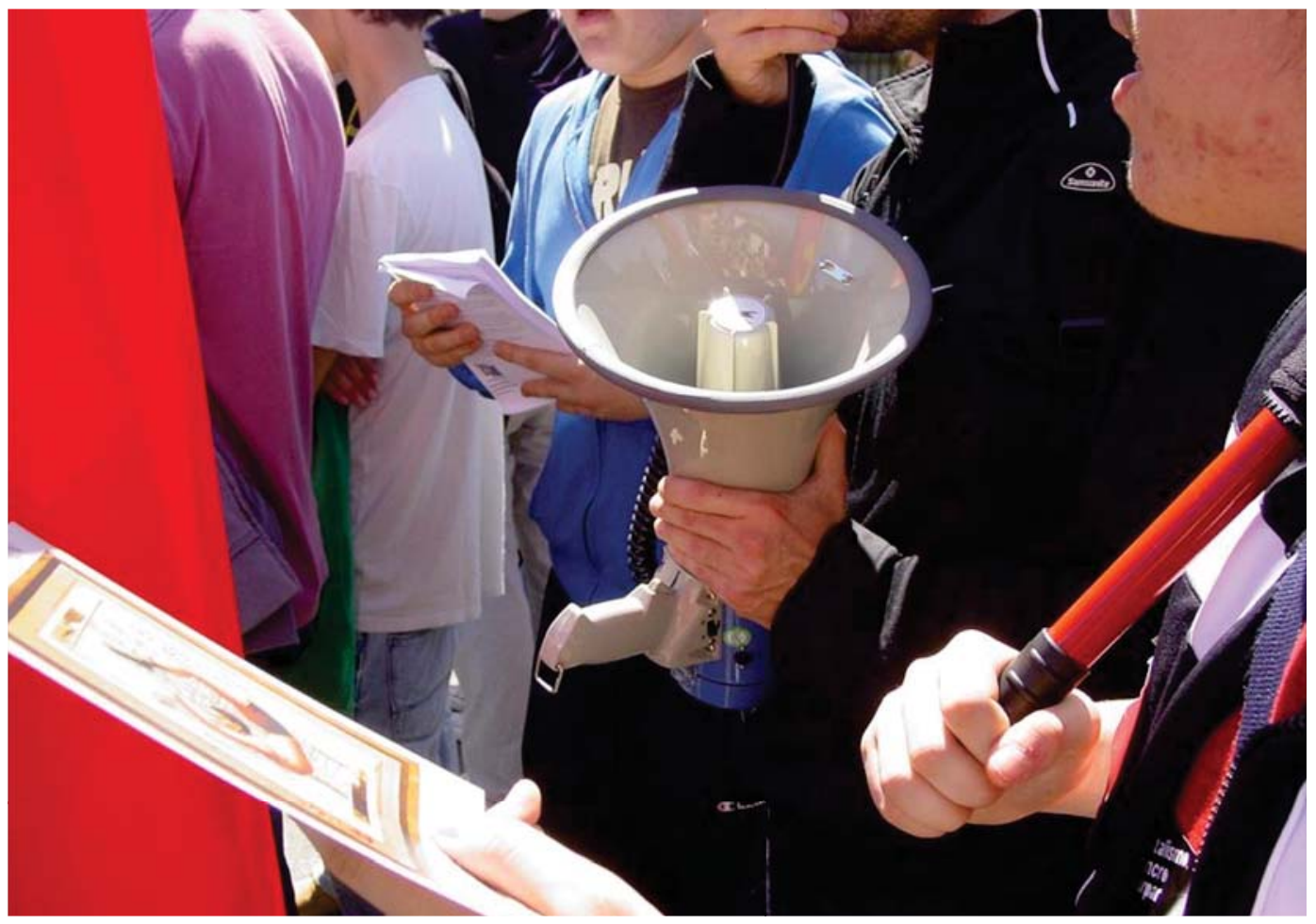

Fig. 7: Postal de "más que nunca", en Santiago de Compostela, España. 
de Internet y grupos sociales. En el año 2012, la convocatoria generó el interés de los educadores de arte de todo el mundo y en el 2013, fueron registradas otras tantas actividades en el boletín de InSEA durante esa semana, lo que demuestra que hubo un impacto significativo del evento en el campo de la educación artística y, lo más importante realmente, fue que las personas fueron capaces de vincular sus pequeñas acciones locales a una escala global, haciéndolas visibles y conectándose a través de las organizaciones mundiales de educación artística en Internet. El evento continúa desarrollándose, y para el actual año 2004, ya existen multitud de iniciativas.

Lo que nos parece sin duda más interesante en este tipo de eventos, no es la fiesta per se, sino las capacidades de las redes (networks) para trabajar en conjunto sobre un determinado tema que los educadores de arte sienten relevante para sus vidas y, donde están dispuestos a invertir algo de su tiempo para hacer visibles las acciones necesarias para promover la educación artística a sabiendas de que, en ese momento y en una gran mayoría de los países, la educación artística no sólo se encuentra subestimada por la sociedad en general sino que se haya en riesgo de desaparición en los currículums de las escuelas.

Quisiéramos destacar aquí el caso del Grupo Activista de Educación con el Arte C3, cuyos miembros abrazaron la oportunidad que les brindaba esta Semana de las Artes para difundir mensajes sobre el valor de la educación artística para la conciencia pública. A través de las redes sociales de educación artística se recogieron un conjunto de palabras sobre el valor de la educación artística. Estas palabras sirvieron de base para el diseño de una postal, con la colaboración de la artista Xesús Carballido, traducido en cinco idiomas, que fue distribuida en Internet a través de diversos grupos sociales de educadores e investigadores que luego hicieron eco del contenido de la misma, imprimiéndola y distribuyéndola por todos los rincones del mundo (Porto Alegre -Brasil ;Porto, Viseu y Lisboa- Portugal; Santiago de Compostela, Madrid y Granada - España; Osaka- Japón).

Al finalizar esta Performance Activista, que llevó el lema "más que nunca", Maria Jesús Agra-Pardiñas, en el nombre de los elementos del grupo, terminó la acción con un nuevo principio:

... No queríamos detener el movimiento que comenzó durante la primera semana de la educación artística internacional!. ... Escribimos las palabras 'educación artística' en la arena para que las ondas puedan transportar el mensaje y llegar a todos los rincones del mundo! (C3).

Retomamos aquí las palabras que citábamos al inicio de este trabajo de McLuhan en aquella entrevista a la revista Playboy: vivimos en un mundo donde es posible estar conectado las veinticuatro horas diarias, provocando encuentros profundos, pero sin embargo, "la aldea tribal global es mucho más separatista -llena de 
combates- que cualquier nacionalismo lo fue alguna vez. El Pueblo es la fisión, no de fusión, a fondo, todo el tiempo" (1996). Por eso, el papel de los educadores del arte es esencial en este medio, ya que pueden usar sus herramientas artísticas y pedagógicas para dar forma a encuentros que pueden transformar la manera en que construimos la noción misma de la educación artística y de la sociedad. Podemos ejercer nuestro papel en las redes y señalar nuevas direcciones para nuestras acciones en un mundo globalizado sujeto a la división y a las fisiones y, en estas fisiones, que mayor oportunidad que prever nuevas formas de educación artística encaminadas a la cohesión social y al desarrollo humano.

\section{Referencias bibliográficas}

Allen, P. et al (2013). Zero Carbon Britain Report: Rethinking the Future. Machynlleth, Powys; Centre for Alternative Technology.

Downes, S. (2012). Connectivism and Connective Knowledge. Retrieved August 12, 2013 from http://www.downes.ca/files/books/Connective_Knowledge19May2012.pdf

Beer, R \& Grauer, K. (2013). Catch and release: Artworks inspiring insight into environmental issues. In: International Journal of Education through Art ,8 (3), pp 349-360.

Fitzgerald, M. (2005). ‘Group Rethink.’MIT Technology Review. June.

Freire, P. (1970). Pedagogia do oprimido. New York: Herder \& Herder.

Giroux, H. A. (1988). Teachers as Intellectuals; Toward a Critical Pedagogy of Learning. New York: Bergin \& Garvey.

Huhmarniemi, M. (2013). Berry wars: A science centre as a forum for a dialogical activist, interdisciplinary art project. In: International Journal of Education through Art, 8 (3). pp 287-303.

Levy, P. (1994). L'intelligence collective. Pour une anthropologie du cyberspace, Paris, La Découverte.

Schuler, D. (2008). Liberating Voices: A Pattern Language for Communications Revolution. MIT Press.

Vaill, P. B., (1996). Learning as a Way of Being. San Francisco, CA, Jossey-Blass Inc. 